\title{
Attunement to haptic information helps skilled performers select implements for striking a ball in cricket
}

\author{
Jonathon Headrick • Ian Renshaw • Ross A. Pinder • \\ Keith Davids
}

Published online: 8 September 2012

(C) Psychonomic Society, Inc. 2012

\begin{abstract}
This study examined the perceptual attunement of relatively skilled individuals to the physical properties of striking implements in the sport of cricket. We also sought to assess whether utilizing bats with different physical properties would influence performance of a specific striking action: the front foot straight drive. Eleven skilled male cricketers (mean age $=16.6 \pm 0.3$ years) from an elite school cricket development program consented to participate in the study. While blindfolded, participants wielded six bats exhibiting different mass and moment of inertia (MOI) characteristics and were asked to identify the three bats they preferred the most for hitting a ball to a maximum distance by performing a front foot straight drive (a common shot in cricket). Next, participants actually attempted to hit balls projected from a ball machine using each of the six bat configurations to enable kinematic analysis of front foot straight drive performance with each implement. Results revealed that, on first choice, the two bats with the smallest mass and MOI values (1 and 2) were most preferred by almost two thirds $(63.7 \%)$ of the participants. Kinematic analysis of movement patterns revealed that bat velocity, step length, and bat-ball contact position measures
\end{abstract}

J. Headrick $(\bowtie) \cdot$ I. Renshaw $\cdot$ R. A. Pinder $\cdot$ K. Davids

School of Exercise and Nutrition Sciences,

Queensland University of Technology,

Victoria Park Road,

Kelvin Grove, Brisbane QLD 4059, Australia

e-mail: headrick.jonathon@gmail.com

J. Headrick $\cdot$ I. Renshaw $\cdot$ R. A. Pinder $\cdot$ K. Davids

Institute of Health and Biomedical Innovation,

Queensland University of Technology,

Musk Avenue,

Kelvin Grove QLD 4059, Australia

\section{R. A. Pinder}

School of Health and Sport Sciences,

University of the Sunshine Coast,

Maroochydore, DC QLD 4558, Australia significantly differed between bats. Data revealed how skilled youth cricketers were attuned to the different bat characteristics and harnessed movement system degeneracy to perform this complex interceptive action.

Keywords Haptics · Perception and action · Goal-directed movements $\cdot$ Degeneracy $\cdot$ Cricket

\section{Introduction}

The ability of humans to determine the utility of tools or objects for undertaking functional behaviors has been studied extensively through manipulating physical properties such as size, shape, and weight, while constraining the visual information available (see Bingham, Schmidt, \& Rosenblum, 1989; Carello, 2004; Solomon \& Turvey, 1988; Turvey, Burton, Amazeen, Butwill, \& Carello, 1998). These investigations are predicated on theoretical insights from ecological psychology on how humans detect information and perceive properties of the environment as affordances during goal-directed behavior (Gibson, 1966, 1979). Gibson (1966) proposed the concept of dynamic touch to highlight the role of the haptic system when detecting information gained through object manipulation (Davids, Bennett, \& Beak, 2002). Dynamic touch refers to the detection of haptic information by the nervous system through mechanoreceptors when tendons, ligaments, and muscles are contorted, extended, or stressed. Research has revealed that haptic information detected through grasping, wielding, hefting, or swinging an implement can be utilized to perceive affordances (i.e., opportunities for action) of an implement in relation to functional task performance (Carello, 2004; Gibson, 1979; Hove, Riley, \& Shockley, 2006; Turvey, 1996; Wagman \& Carello, 2003).

To understand the role of dynamic touch in perceiving affordances of implements, experimenters have occluded the 
vision of participants to negate the use of visual information in object selection (Amazeen \& Turvey, 1996; Michaels, Weier, \& Harrison, 2007). This methodological manipulation forces participants to rely on haptic information detected from wielding an implement to perceive its affordances for performing a designated action, rather than visually assessing length, shape, and size characteristics. Physical or mechanical properties of an implement perceived during wielding include its mass and resistance to rotation, or moment of inertia (MOI) (Shockley, Carello, \& Turvey, 2004; Wagman \& Carello, 2001). Together, these variables refer to how easily an implement can be moved from a resting state with regard to its overall mass and the distribution of that mass. Hence, the mass and MOI properties of an implement can influence how a person perceives its suitability for a particular task, such as hitting a ball, depending on interactions with personal constraints, such as physical strength, limb length, previous experience, and skill, as well as specific task goals (Newell, 1986). In respect to the task of actually striking an object such as a ball, perceiving the location of the center of percussion (COP), or "sweet spot" of an implement is also influential in perceiving its suitability for an interceptive action (Carello, Thuot, Anderson, \& Turvey, 1999; Fisher, Vogwell, \& Ansell, 2006). The COP refers to the point of impact on a bat that results in minimal vibration through the hand(s) holding the bat, which can also be detected from the haptic information about the distribution of mass and length of the bat, gained through wielding prior to striking a ball (Carello, Thuot, \& Turvey, 2000).

In order to select a tool or implement that offers affordances for completing a specific task, participants must exhibit perceptual attunement to the physical properties of the tool that make it suitable for the task. Perceptual attunement refers to an individual's learned ability to detect key information for a given task that has the potential to influence emergent decision-making behaviors (Araújo, Davids, \& Hristovski, 2006; Fajen, Riley, \& Turvey, 2009; Weast, Shockley, \& Riley, 2011). Expert or skilled performers in sport are deemed to display attunement to specific perceptual variables relating to a task because of extensive amounts of specific task experience and practice (Smith, Flach, Dittman, \& Stanard, 2001). For example, hockey players studied by Hove et al. (2006) perceived the affordances of hockey sticks for power and precision tasks differently than those participants who were not hockey players. These findings suggested that, when wielding hockey sticks with novel physical properties, skilled hockey players revealed that they were attuned to different, more functionally specific information, as compared with a sample of less skilled hockey players. Despite these studies of perceptual attunement, there have been few attempts to examine performance of specific actions with implements selected on the basis of haptic information.

Individuals who display perceptual attunement to key informational variables have the ability to flexibly adapt their behaviors when dynamic performance circumstances are changed or the constraints of a task are manipulated (Fajen et al., 2009). In other words, skilled or attuned performers find novel strategies for achieving task goals when aspects of the performance environment change. The term degeneracy has been used to describe how structurally different elements of neurobiological systems are able to produce the same output across variable performance contexts (Edelman \& Gally, 2001). Through inherent processes of self-organization, degenerate neurobiological systems (e.g., performers in sport) undergo phase transitions, leading to emergent behavior patterns that harness affordances offered by the environment to achieve a desired function or outcome (Davids \& Araújo, 2010; Kelso, 1995; Rein, Davids, \& Button, 2009). Therefore, a skilled performer confronted by fluctuating constraints would be expected to adapt their behaviors to achieve performance objectives through their perceptual attunement to task-specific informational variables (i.e., haptic information).

Studies of implements with different physical characteristics have often focused on fundamental behaviors such as lifting and reaching (e.g., Solomon \& Turvey, 1988; Turvey, et al., 1998). However, similar methods have infrequently been applied to the study of dynamic, multiarticular interceptive actions in sport performance contexts. Some previous work has demonstrated the sensitivity of children and adults to haptic information of tennis rackets with the same mass, but with different inertial characteristics (Beak, Davids, \& Bennett, 2000; Davids et al., 2002). Six weighted rackets were wielded by children, inexperienced adults, and experienced adults in both visual and nonvisual conditions. Each participant ranked their three preferred rackets for hitting a ball to a maximum distance. Findings revealed that each group showed sensitivity to changes in racket characteristics, with the children favoring rackets with smaller MOI, as compared with the two adult groups, in both visual and nonvisual conditions. Unfortunately, the study of Beak et al. (2000) did not actually require participants to hit tennis balls. Therefore, it is still unknown whether the perception of controllability of a racket, as affected by the racket's mass distribution in relation to the effective point of rotation, was scaled to individual characteristics or was functional for the performance of a specific action (see Shockley et al., 2004; Shockley, Grocki, Carello, \& Turvey, 2001). Hence, it is unclear whether the perceived affordances and attunement of participants corresponded with functional performance (task) outcomes.

Biomechanical analyses have revealed how the physical properties of implements affect swing characteristics and velocity in interceptive sports actions such as hitting in baseball and softball (e.g., Cross \& Bower, 2006). Bat swing speeds were found to decrease when the mass and MOI of modified bats and weighted rods (simulating bats) were increased (Koenig, Mitchell, Hannigan, \& Clutter, 2004). Swing patterning was also found to vary when bats of 
different mass and MOI characteristics were used as part of a baseball warm-up, revealing again that the bats with the greatest mass and MOI produced slower swing speeds (Southard \& Groomer, 2003). Furthermore, baseball and softball bat MOI has been found to be more influential than bat mass for changing swing characteristics, as evidenced by linear correlations between swing velocity and both bat mass and MOI (Fleisig, Zheng, Stodden, \& Andrews, 2002). These findings exemplify how the mass and MOI of baseball/softball bats together influence swing characteristics during interceptive hitting tasks.

\section{Overview of cricket batting}

Cricket batting is a sport performance context that involves the interception of a moving ball with a handheld implement (a cricket bat; see Fig. 1). Such actions are worthy of study because they can provide significant insights into the control of human behavior under changing task constraints (Davids, Renshaw, \& Glazier, 2005). Bats are used as an implement to intercept a ball delivered by bowlers at varying speeds and bounce points and a range of flight characteristics (e.g., spin, swing). Depending on the type of delivery bowled at the batter, a bat may be swung in highly specific ways to perform particular strokes when defending the stumps from the ball (e.g., back foot and front foot defense) or to attack the delivery with the intention of scoring runs (e.g., drives, pulls, and hooks). It is important to note that, when specific cricket strokes are performed, the bat needs to be swung in specific displacement trajectories, differing in planes of motion. For example, the front foot drive involves a bat swing in the sagittal plane, whereas the pull shot typically involves the bat being swung in the horizontal (transverse) plane on the back foot. Preferences for bat selection are individualized depending on individual constraints such as playing style (e.g., aggressive or conservative), body proportions, and muscular strength. Bats may vary in size, mass, and profile/shape, all of which may affect the perceived heaviness and suitability for each individual (Shockley et al., 2004). Hence, haptic information plays a significant role in attempting to select a bat that affords opportunities to effectively perform cricket shots such as front foot straight drives.

The front foot straight drive was selected as the action component in this study of dynamic touch in cricket batting because it is an extension of the most common stroke in cricket, the front foot defense (Pinder, Davids, Renshaw, \& Araújo, 2011; Stretch, Buys, Du Toit, \& Viljoen, 1998). For this reason, it has been extensively studied in previous research and is also suitably planar to allow for two-dimensional kinematic analyses of performance (Stretch et al., 1998). Typically, the front foot drive is used to hit the ball along the ground to minimize the chance of it being caught by a fielder, although the ball can also be lofted with this stroke (Woolmer, Noakes, \& Moffett, 2008). Measures such as bat velocity, step length, and body segment angles have all provided insights into how cricket bat-ball interceptive actions are coordinated and have been used to compare successful and unsuccessful performances of shots (Stretch, Bartlett, \& Davids, 2000; Stretch et al., 1998; Woolmer et al., 2008).

\section{Aims and objectives}

Our first objective in this study was to establish whether preferences, based on haptic perception of the mechanical properties of cricket bats for performing a front foot straight drive, were evident in a sample of skilled youth participants. The second objective was to investigate whether bats with different physical properties actually constrained movement kinematics of the same participants when they performed the front foot straight drive shot in cricket. Consideration of both aims allowed us to answer two key questions: Were skilled participants attuned to the properties of cricket bats allowing them to perceive the functionality of bats for performing a specific stroke in cricket, in the form of haptic information detected through wielding? And, how did the same participants utilize different bats for performing a front foot straight drive with the intention to straight drive a ball to a maximum distance? On the basis of some previous work, it was hypothesized that participants would show individualized preferences when wielding some or all of the bats, similar to previous
Fig. 1 Representation of weight positions with corresponding bat characteristics and measurements for each of the six bat configurations (not to scale)

\begin{tabular}{lcccccc}
\hline Bat & $\begin{array}{c}\text { Weight strip } \\
\text { position }\end{array}$ & $\begin{array}{c}\text { Mass } \\
(\mathbf{k g})\end{array}$ & $\begin{array}{c}\text { Mass } \\
(\mathbf{l b} / \mathbf{o z})\end{array}$ & $\begin{array}{c}\text { Average } \\
\text { swing time } \\
\text { (s) }\end{array}$ & $\begin{array}{c}\text { Balancing } \\
\text { Point from } \\
\text { pivot point }(\mathbf{m})\end{array}$ & $\begin{array}{c}\text { MOI about } \\
\text { pivot point } \\
\left(\mathbf{k g ~ m}^{2}\right)\end{array}$ \\
\hline $\mathbf{1}$ & No weights & 1.050 & $2 / 5.03$ & 1.322 & 0.389 & 0.177 \\
$\mathbf{2}$ & $7-8$ & 1.178 & $2 / 9.55$ & 1.405 & 0.389 & 0.199 \\
$\mathbf{3 a}$ & $7-9$ & 1.242 & $2 / 11.81$ & 1.425 & 0.381 & 0.205 \\
$\mathbf{4 a}$ & $1-3$ & 1.242 & $2 / 11.81$ & 1.515 & 0.426 & 0.229 \\
$\mathbf{3 b}$ & $5-9$ & 1.370 & $3 / 0.32$ & 1.445 & 0.393 & 0.234 \\
$\mathbf{4 b}$ & $1-5$ & 1.370 & $3 / 0.32$ & 1.518 & 0.429 & 0.255 \\
\hline
\end{tabular}

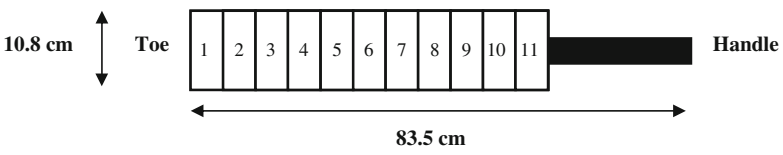


observations in the sport of tennis where rackets with identical mass, but a smaller MOI, were preferred by young children, while rackets with a greater MOI were preferred by adults (Beak et al., 2000; Davids et al., 2002). On the basis of movement system degeneracy, it was also expected that varied kinematic patterns would be observed when front foot straight drive performances were compared for bats with comparatively small and large mass and MOI values. Specifically, bats with a greater mass and MOI were expected to return slower swing velocities. Subsequently, it was anticipated that if a bat was most preferred by a participant during the task of wielding for the purposes of selecting an implement to perform a front foot drive, this selection preference would be confirmed through associated kinematic measure(s) observed during actual performance of that particular cricket stroke.

\section{Method}

\section{Participants}

Eleven male (age $=16.6 \pm 0.3$ years) participants $(9$ righthanded, 2 left-handed) from a local school cricket development program provided informed written consent to participate in the study after ethical clearance was obtained through a university ethics committee. Participants reported competitive playing experience of $7.5 \pm 0.5$ years and were deemed to be skilled, at the control stage of Newell's (1985) model of motor learning, by two level 3 cricket coaches and motor learning specialists. Participants at the control stage of learning were preferred over novices, since they had a functional understanding of the task requirements and previous experience in selecting suitable bats (Weast et al., 2011). All participants were familiar with the testing facility and equipment through their participation in the school's cricket development program.

\section{Setup/apparatus}

A small men's cricket bat (Gabba sporting products, Brisbane), $83.5 \mathrm{~cm}$ in length, maximum blade width of $10.8 \mathrm{~cm}$, and mass of $1.05 \mathrm{~kg}$, was selected as the base test bat due to its relatively low mass and generic characteristics. To manipulate the bat's mass and inertial properties (simulating bats of different characteristics), flat weights in the form of coins $(0.064 \mathrm{~kg})$ were attached to the back of the bat, comparable to the $0.05-\mathrm{kg}$ external weights added by Beak et al. (2000) and Davids et al. (2002) in tennis. Through pilot work, single weights were deemed insufficient to clearly distinguish between bats. Therefore, pairs of weights (total of $0.128 \mathrm{~kg}$ ) were attached either side of the spine of the bat. Figure 1 details the position of the weights for the six bat configurations, which included two lighter, balanced bats $(1,2)$, two "top-heavy" bats $(3 \mathrm{a}, 3 \mathrm{~b})$, and two "bottom-heavy" bats $(4 a, 4 b)$. The selected bat mass configurations represented a range of bat types commonly used in cricket batting performance by the youth participants in this study. Participants were naive as to the specific aims of the experiment and did not reveal any awareness of bat differences based on positioning of the weights.

To determine the MOI of the different bat configurations, the time taken for each bat to complete a single pendulum motion was measured (average from ten trials), with the bat suspended from a pivot point 6 in. $(15.2 \mathrm{~cm})$ from the end of the handle (ASTM standard) (Fleisig et al., 2002). The equation below was then used to identify the MOI $(I)$, where $T=$ pendulum swing time (s), $m=$ bat mass (kg), $g=$ acceleration due to gravity $\left(\mathrm{m} \cdot \mathrm{s}^{-1}\right)$, and $d=$ distance from balance point to pivot point (bat characteristics are listed in Fig. 1):

$I=T^{2} m g d / 4 \pi^{2}$.

\section{Wielding task}

The wielding task required participants to wear their own batting gloves and a blindfold before being handed the six bat configurations in random order. Participants were asked to identify their three most favored bats perceived to be most functional for performing a front foot straight drive with the intention of striking a cricket ball to a maximum distance. Each bat was placed in the bottom hand of each participant by a research team member before being wielded/swung (by the handle only) in any manner with either or both hands for as long as needed. Once all bats had been wielded, each participant had the option to wield any of the bats again, before being asked to list their three preferred bat numbers in descending order. No balls were hit during this perceptual judgment task.

\section{Hitting task}

The hitting task required participants to front foot straight drive balls ("Oz" machine balls) projected (release height $0.85 \mathrm{~m}$ ) from a projection machine (Winters Solutions "Devon Trainer", Highfields, Queensland) positioned $17 \mathrm{~m}$ from the participant's stumps, or approximately $15.5 \mathrm{~m}$ from the participant. Positioning of the ball machine was determined through pilot work to allow for a slow projection speed $\left(11.3 \pm 0.4 \mathrm{~m} \cdot \mathrm{s}^{-1} \sim 40 \mathrm{~km} \cdot \mathrm{h}^{-1}\right)$ while maintaining conventional ball flight and bounce characteristics (i.e., no excessive loop or bounce) to land the ball in a position suitable for a front foot straight drive. The ball machine was used to control and standardize the ball delivery characteristics, with a slow speed chosen to negate the importance of prerelease information available from a bowler's actions (Pinder, Renshaw, \& Davids, 2009; Renshaw, Oldham, Davids, \& Golds, 2007). All participants had experience of practicing against the ball machine and were required to wear full protective equipment. Contrasting markers were placed on 
the: helmet (temple), knees (approximate rotation point on the pad), feet (proximal phalanx of the hallux), and bat (outside edge of the toe/end). To capture the displacement of these selected points during performance, a Sony (HVR-V1P) video camera $(100 \mathrm{~Hz}, 1 / 300$ shutter speed) was positioned $8 \mathrm{~m}$ from the participant, orientated perpendicular to the action (side on). Participants were presented with the six bats in random order (different from the wielding task) and were required to perform front foot straight drives, attempting to achieve maximum hitting distance. No specific instructions were given regarding how to perform the front foot straight drive or whether the ball should be hit along the ground or in the air. Three trials with each bat, which were deemed to exhibit a high quality of bat-ball contact (i.e., hitting the center of the bat face), were recorded for analysis. Quality of interceptive contact was determined live by an Australian level 3 coach operating the ball machine and later confirmed through video analysis (see Müller \& Abernethy, 2008).

\section{Analysis}

Data on bat choice rankings for each participant in the wielding task were collated and displayed in a frequency plot to display variance in bat choice. Paired-sample correlation tests were performed to determine the influence of both mass and MOI, on the frequency of first choices and total number of choices (first, second, and third choices combined) in bats. The hitting task produced 198 trials that were subsequently digitized using Vicon Motus software (Vicon Motion Systems, U.K.). Following previous research, step length, head-front-knee-foot angle (at contact), head-to-point-of-contact horizontal distance, and bat end point velocity (contact and maximum) were identified as dependent variables (Stretch et al., 2000; Stretch et al., 1998; Woolmer et al., 2008). Data from dependent measures were compared for each bat configuration using a one-way repeated measures analysis of variance (ANOVA) with pairwise comparisons (alpha level <.05). Bonferroni corrections were used to control for Type 1 errors, and the Huynh-Feldt method was employed to correct for violations of the sphericity assumption in the repeated measures design (Field, 2009).

\section{Results}

\section{Wielding task}

Results from the wielding task (see Fig. 2) revealed that, in this sample of participants, bat 1 was the most popular first choice (45.5\%), followed by 2 and 4a (18.2\%). Therefore, the two bats with the smallest MOI and mass values (1 and 2) were most preferred on first choice by almost two thirds $(63.7 \%)$ of the participants. When first, second, and third

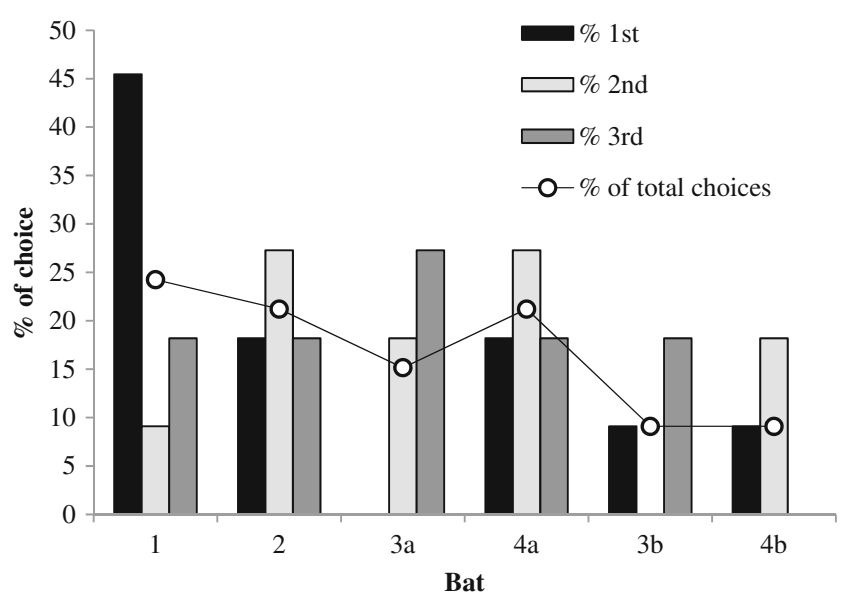

Fig. 2 Percentages of choices for first, second, and third preferred bats and total accumulative choices in the blindfolded wielding task

choices were combined, bat 1 was again the most preferred, with $24.2 \%$ of total choices. A significant negative correlation was found between bat mass and total choices $r(4)=.92$, $p<.01$. Mass with first choice (.79), MOI with first choice (.63), and MOI with total choices (.79) all returned negative correlations that were not statistically significant.

Hitting task

Results from the hitting task are presented in Table 1. In terms of movement kinematics, a significant difference was observed in step lengths between bat configurations, $F(4.3,138.5)=4.14$, $p<.05$. Pairwise comparisons revealed that step lengths were shorter with bat 1 than with 2 and 3a. The alignment of the head in relation to the bat-ball contact point also returned statistically significant differences, $F(3.7,116.9)=7.92, p<.05$. Bat-ball contact points for all bats were found to occur out in front of the head position. However, pairwise comparisons revealed that the contact points with bats 1,2, and 3a were significantly further out in front of the head position than when both $3 b$ and $4 a$ were used. In terms of maximum velocity of stroke performance, differences were observed between bats, $F(3.9,126.3)=7.41$, $p<.05$. Bats $1,2,3 \mathrm{a}$, and $3 \mathrm{~b}$ all displayed significantly faster maximum velocities during stroke performance than did $4 \mathrm{~b}$. Bat 1 was also found to have a significantly faster maximum velocity than did $4 \mathrm{a}$. Bat velocity at point of contact with the ball was significantly constrained by different bat configurations, $F(5,27)=3.7, p<.05$, with pairwise comparisons revealing that $4 \mathrm{~b}$ produced a significantly slower velocity, as compared with 3a. All differences were significant at the $p<.05$ level.

Figure 3 displays exemplar kinematic results for participants 1 and 8 so that the strategies or techniques that individual participants used to complete the task with each bat can be compared. During the wielding task, participant 1 
Table 1 Hitting task kinematic measures results (post hoc significant differences $[p<.05]$ between bats indicated by matching*)

\begin{tabular}{|c|c|c|c|c|c|c|}
\hline Bat & 1 & 2 & $3 a$ & $4 a$ & $3 b$ & $4 \mathrm{~b}$ \\
\hline Step length (m) & $0.64 \pm .16^{*, * *}$ & $0.71 \pm .14^{*}$ & $0.72 \pm .14^{* *}$ & $0.68 \pm .17$ & $0.68 \pm .16$ & $0.68 \pm .14$ \\
\hline $\begin{array}{l}\text { Head-knee-front-foot } \\
\text { angle at contact (degrees) }\end{array}$ & $180 \pm 10$ & $180 \pm 10$ & $177 \pm 11$ & $179 \pm 10$ & $178 \pm 10$ & $179 \pm 12$ \\
\hline $\begin{array}{l}\text { Head-contact point, } \\
\text { horizontal distance (m) }\end{array}$ & $0.54 \pm .17^{*}$ & $0.53 \pm .18^{* *}$ & $0.52 \pm .15^{* * *}$ & $0.42 \pm .12^{*, * *, * * *}$ & $0.38 \pm .13^{*, * *, * * *}$ & $0.45 \pm .10$ \\
\hline $\begin{array}{l}\text { Maximum bat } \\
\text { velocity }\left(\mathrm{ms}^{-1}\right)\end{array}$ & $11.25 \pm 1.28^{*, * * * *}$ & $10.89 \pm 1.53^{* *}$ & $11.03 \pm 1.20^{* * *}$ & $10.52 \pm 1.17^{* * * *}$ & $10.97 \pm 1.32^{* * * * * *}$ & $10.19 \pm .86^{*, * *, * * *, * * * * *}$ \\
\hline Bat velocity at contact $\left(\mathrm{ms}^{-1}\right)$ & $9.82 \pm 1.38$ & $9.77 \pm 1.92$ & $9.97 \pm 1.53^{*}$ & $9.79 \pm 1.4$ & $10.13 \pm 1.59$ & $9.48 \pm 1.01^{*}$ \\
\hline
\end{tabular}

(left) chose bat 2 as the most preferred bat, and participant 8 chose bat 1 (right). These figures exemplify key kinematic findings reported in Table 1 , such as the shorter step lengths (Fig. 3a) and higher maximum (3d) and contact (3e) velocities when bat 1 was used. The individualized strategies for performing the hitting task are evident by observing the variability between these 2 participants - in particular, the head-knee-foot angles in Fig. 3 b.

\section{Discussion}

The aims of the study were twofold. First, we sought to establish the existence of attunement in skilled youth cricketers to the affordances offered by bats of varied physical properties in a blind wielding task. Second, we aimed to investigate whether the same bats constrained the emergent kinematics of performing a front foot straight drive shot for each participant. Our results revealed that participants did display attunement, in the form of preferences for the physical properties of bats they perceived most functional for performance of the interceptive action. We also observed how the emergent behaviors of the participants varied between bats through the identification of significant variations in kinematic performance measures. These findings have implications for understanding the perceptual attunement of skilled individuals to the haptic information available from handheld implements as tools for action. Furthermore, participants demonstrated perceptual-motor system degeneracy by displaying diverse strategies for completing a hitting task when constrained by bats of different physical characteristics.

\section{Wielding task}

Results for the haptic wielding task revealed varied preferences for bat characteristics in participants; however, typically, the bats with the smallest mass and MOI $(1,2)$ were most preferred, with $63.7 \%$ of first choices. Moreover, the two bats $(3 \mathrm{~b}, 4 \mathrm{~b})$ with the greatest mass and MOI were least favored across all choices. The findings indicate that the majority of participants perceived that the affordances offered by bats with the smallest mass and MOI values were most functional for performing a front foot straight drive with the aim of achieving maximal distance. Therefore, as has also been reported in the context of tennis (Beak et al., 2000; Davids et al., 2002), our participants who were at the control stage of learning were attuned to the physical properties of handheld ball-striking implements. The perceptual attunement of participants was demonstrated by the clear preferences for the haptic information offered by bat 1 in particular, which suggests that the affordances offered by this bat were well suited to the task. Furthermore, participants were found to discriminate between bats on the basis of their mass and MOI properties. A significant negative correlation was found between bat mass and the total frequency of bat choices. This finding highlights the influence of overall bat mass on choices made by the participants. However, data from the wielding task also suggested that MOI influenced choices. For example, bats $3 \mathrm{a}$ and $4 \mathrm{a}$ were the same mass but differed in MOI characteristics, which may account for the different bat choice results (see Fig. 2). Alternatively, bats $3 \mathrm{~b}$ and $4 \mathrm{~b}$, which also had different MOI values from the same overall mass, displayed very similar bat choices, suggesting that their shared high mass influenced the choices (or lack thereof) made in the wielding task.

\section{Hitting task}

\section{Step length}

Step length has been documented as a key determinant of balance and the transfer of weight during performance of a front foot straight drive, therefore influencing the characteristics of the bat swing (Stretch et al., 1998). The step lengths reported in this study were found to be similar to those found for the front foot drive by Stretch et al. and, overall, slightly shorter than the values reported by Pinder et al. (2009), possibly as a result of the different task instructions. Results from the hitting task in our study revealed that using bats with different physical properties influenced the length of the step taken by participants. In particular, step length 
Fig. 3 Exemplar kinematic results for participant 1 (left) (first choice, bat 2) and participant 8 (right) (first choice, bat 1): a step length, b head-knee-foot angle at contact, $\mathbf{c}$ head-ball position at contact, $\mathbf{d}$ maximum bat velocity, e bat velocity at contact
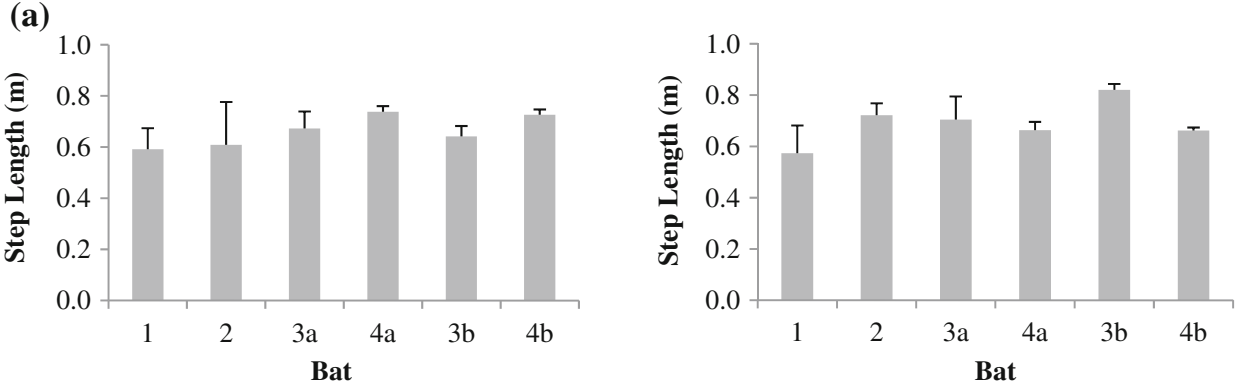

(b)
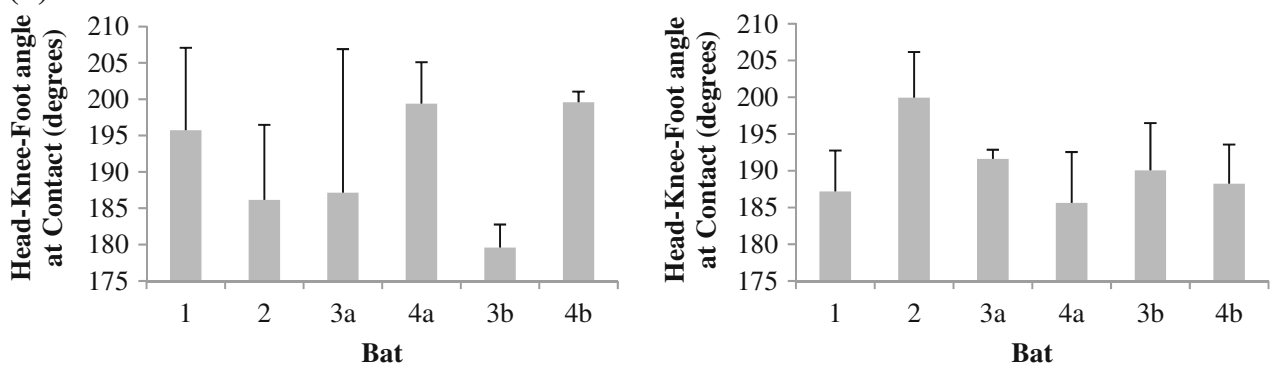

(c)
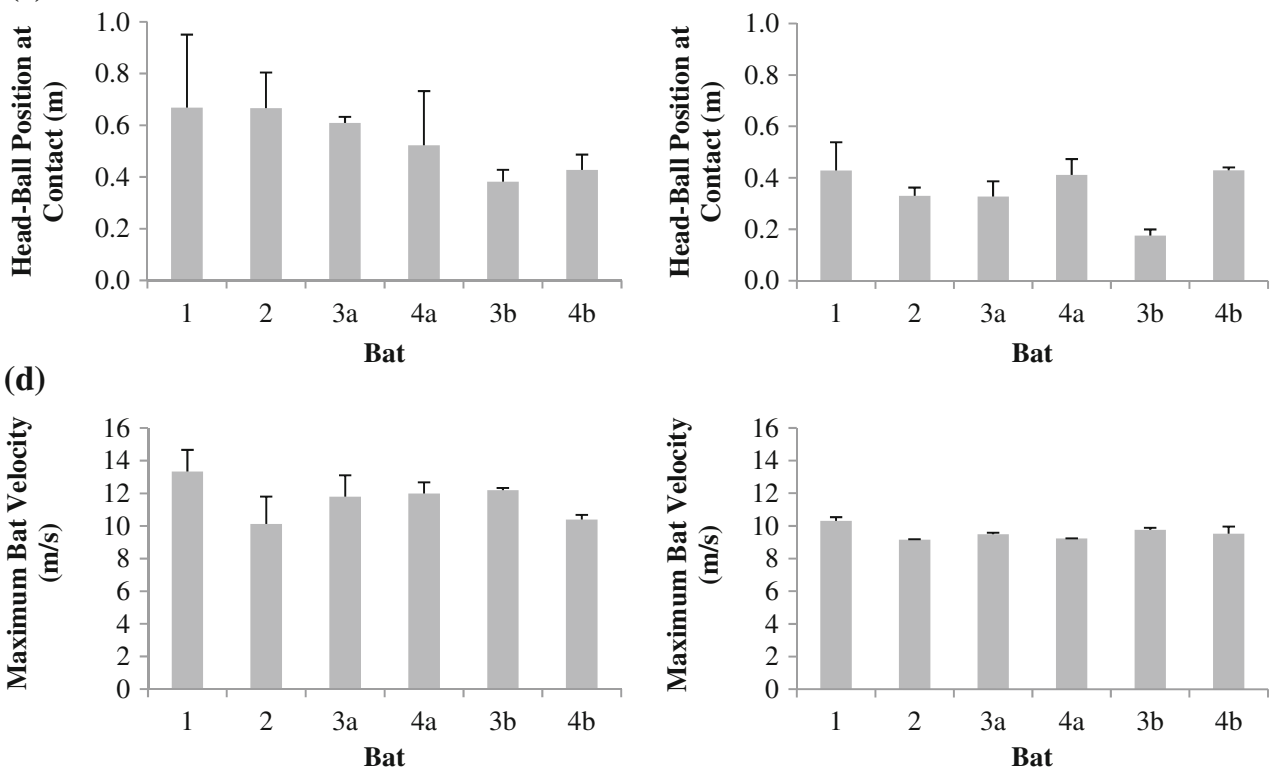

(e)

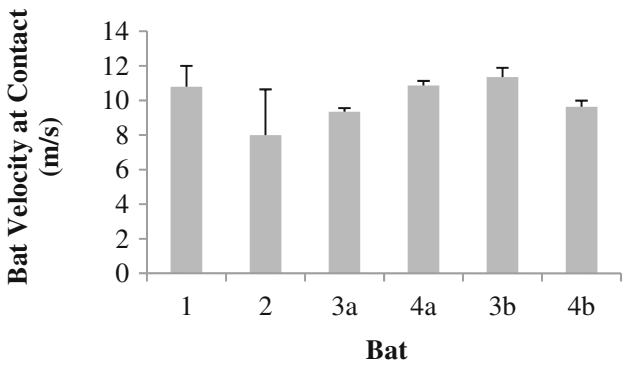

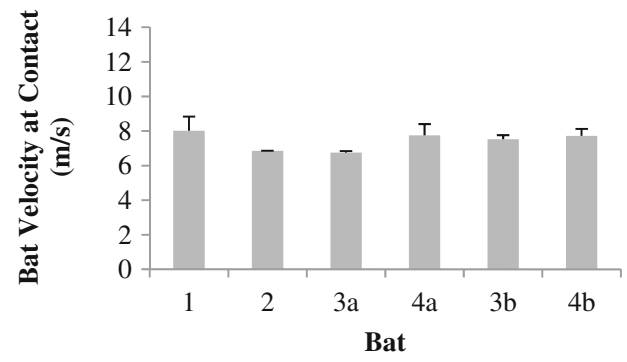

values were found to be smallest for trials using bat 1 and statistically different from the longer step lengths observed when the same participants used 2 and $3 a$. These data reveal how bats with different physical properties constrained the emergence of action in participants. Overall, the longest step lengths were recorded using bat 2 and $3 \mathrm{a}$, which were the two bats with the weights concentrated closest to the handle end. The longer step lengths observed with these two bats (and to a lesser extent, bats 3b, 4a, and $4 \mathrm{~b}$ ) suggested that, in order to hit the ball a maximum distance using bats with 
greater mass and MOI, each participant adopted lengthened preparatory movements and, consequently, swing durations, in contrast to fast compact swings with the lighter bat 1 .

\section{Contact point}

Contrary to previous observations that the contact point occurred in close alignment to the position of the head or front foot (Elliott, Baker, \& Foster, 1993; Stretch et al., 1998), in this study, bat-ball contact points were found to occur well out in front of the position of the head for all bat configurations (see Table 1 and Fig. 3c). When participants were using the bats with the lightest mass (1 and 2) and those with the additional mass concentrated closer to the handle $(2,3 a)$, more of the swing was completed before contacting the ball. Figure $3 \mathrm{c}$ shows that the individual performance characteristics of participant 8 (right) slightly contradicted this finding, with bats $4 \mathrm{a}$ and $4 \mathrm{~b}$ displaying similar distances to bat 1 . Bats $3 \mathrm{a}$ and $4 \mathrm{a}$, which shared the same mass but differed in MOI, were found to display significantly different contact points during the hitting task. ${ }^{1}$ This finding highlights how the MOI of bats can influence aspects of performance away from the influence of variable mass. Overall, contact points for bats 1,2, and 3a all occurred significantly further in front of the head, which suggests that the ball was hit earlier in its flight and was more likely to be hit in the air, as compared with both $3 \mathrm{~b}$ and $4 \mathrm{a}$. Significant findings for step length and bat velocity results indicated that these three bat configurations $(1,2$, and $3 a)$ in particular substantially influenced the performance of the front foot straight drive. A likely reason for the difference in these results is the instructional constraint in our study to hit the ball with the intention of achieving maximum distance, rather than simply performing a front foot drive. Therefore, contrary to most cricket practice methods, participants were not constrained by the need to hit the ball along the ground.

\section{Bat velocity}

All maximum bat velocity values were found to occur before the point of contact, which is in agreement with previous studies of cricket stroke performance (e.g., Stretch et al., 1998). As hypothesized from the findings of previous studies (e.g., Cross \& Bower, 2006; Koenig et al., 2004; Southard \& Groomer, 2003), the bat with the equal highest mass and greatest MOI $(4 \mathrm{~b})$ produced the slowest velocity at contact. The velocity of bat $4 \mathrm{~b}$ was significantly slower than that of $3 \mathrm{a}$, but not $3 \mathrm{~b}$ (highest mean velocity),

\footnotetext{
${ }^{1}$ The potential influence of COP (e.g., Carello et al., 2000) was found to be minimal, since COP values were comparable for all bats: bat 1 , $0.433 \mathrm{~m}$; bat $2,0.434 \mathrm{~m}$; bat $3 \mathrm{a}, 0.433 \mathrm{~m}$; bat $4 \mathrm{a}, 0.433 \mathrm{~m}$; bat $3 \mathrm{~b}$, $0.435 \mathrm{~m}$; bat $4 \mathrm{~b}, 0.434 \mathrm{~m}$.
}

due to greater variability between individual participants and trials, as evidenced by the standard deviation data (see Table 1). Nevertheless, these values demonstrated how two bats of equal mass $(3 \mathrm{~b}, 4 \mathrm{~b})$ can produce different emergent performance outcomes in a dynamic interceptive action due to varied MOI, as evidenced in Fig. 3e (left). Maximum bat velocity values also revealed $4 \mathrm{~b}$ to be the slowest, followed by the other "bottom heavy" bat, 4a. Bat 1 produced the fastest maximum swing velocity, but not the fastest contact velocity. This finding suggests that participants needed to slow down their swing to achieve high-quality bat-ball contact.

\section{Importance of instruction}

The variable techniques for performing a front foot straight drive with each different bat can be attributed to the generic instructions given to the participants, as well as the interaction between unique personal constraints and the different physical properties of bats. Participants were left to decide for themselves how to strike the ball using a front foot straight drive, with no specific instructional constraints on technique or a requirement to hit the ball along the ground. As a result, different patterns of behavior emerged when bats of different physical characteristics were used. However, similar performance outcomes were achieved. Participants were observed to display system degeneracy, whereby the perceived affordances of each bat resulted in the emergence of different kinematic patterns and strategies (see Fig. 3) in order to achieve the same performance outcome (Edelman \& Gally, 2001; Rein et al., 2009). Furthermore, variations in emergent behaviors during the hitting task revealed that the skilled youth participants in this study were able to adapt or recalibrate (see Fajen, Diaz, \& Cramer, 2011) their movement patterns in response to the affordances offered by different bat characteristics, while still achieving the prescribed task objectives.

Implications

A major theoretical implication from this study is that the physical properties of striking implements like cricket bats affect the perceptual information detected by skilled youth participants at the control stage of learning to regulate batting actions. Participants were found to display perceptual attunement to haptic information of bats differing in physical properties, as evidenced through preferences in bat selection. These findings are consistent with those from previous investigations of implement selection in sport interceptive actions (Beak et al., 2000; Davids et al., 2002; Hove et al., 2006). However, we contributed to understanding in this area by demonstrating that most skilled participants in this specific study selected the bats with smaller mass and 
MOI when swinging a preferred cricket bat, in relation to the performance of a front foot straight drive. Additionally, during the hitting task, we found participants displayed system degeneracy by adopting subtly different emergent strategies or techniques to fulfil the task when constrained by the affordances offered by each bat configuration.

Limitations and future directions

An interesting finding was that changing bat characteristics led to reorganization in the coordination of the front foot straight drive. Future research should examine how manipulating other bat properties - for example, length, handle thickness, and center of percussion-may influence how participants perceive a bat's affordances for performing interceptive actions. Further investigations should also aim to establish whether preferences in bat characteristics are evident for other cricket shots, particularly horizontal strokes (e.g., pull or hook shot) that require different movement organization to swing the bat in fundamentally different planes of motion. Therefore, future work could identify whether a particular type of cricket stroke is most functional for assessing the haptic information of bats, as opposed to general swinging, which does not relate to actually hitting a cricket ball. Additionally, three-dimensional analysis would provide greater depth of kinematic information about the performance of cricket shots with different bats.

\section{Conclusions}

As was predicted, participants were found to display varied preferences and kinematic responses when performing cricket shots with differently configured bats. Bats with greater mass and MOI were found to return slower swing velocities. However, somewhat unexpectedly, the bat with the smallest mass and MOI produced the shortest step length, along with the fastest maximum velocity. The skilled youth participants were observed to show perceptual attunement to the affordances offered by haptic information of bats with varied physical properties. While performing interceptive actions, participants were also found to display system degeneracy by adopting novel emergent behavior patterns to strike a ball the furthest distance when constrained by the different bats. Overall, this investigation exemplifies how skilled performers are perceptually attuned to haptic information of handheld implements for the completion of complex interceptive actions.

Author Note This project was supported by a grant from the Queensland University of Technology. The authors would like to recognize the support of Darren Holder and the Brisbane Grammar School cricket program in conducting this study.

\section{References}

Amazeen, E. L., \& Turvey, M. T. (1996). Weight perception and the haptic size weight illusion are functions of the inertia tensor. Journal of Experimental Psychology-Human Perception and Performance, 22(1), 213-232. doi:10.1037/0096-1523.22.1.213

Araújo, D., Davids, K., \& Hristovski, R. (2006). The ecological dynamics of decision making in sport. Psychoogy of Sport and Exercise, 7, 653-676. doi:10.1016/j.psychsport.2006.07.002

Beak, S., Davids, K., \& Bennett, S. (2000). One size fits all? Sensitivity to moment of inertia information from tennis rackets in children and adults. In S. J. Haake \& A. O. Coe (Eds.), Tennis science \& technology (pp. 109-117). London: Blackwell.

Bingham, G. P., Schmidt, R. C., \& Rosenblum, L. D. (1989). Hefting for a maximum distance throw: A smart perceptual mechanism. Journal of Experimental Psychology: Human Perception and Performance, 15(3), 507-528. doi:10.1037/0096-1523.15.3.507

Carello, C. (2004). Perceiving affordances by dynamic touch: Hints from the control of movement. Ecological Psychology, 16(1), 3136. doi:10.1207/s15326969eco1601_4

Carello, C., Thuot, S., Anderson, K. L., \& Turvey, M. T. (1999). Perceiving the sweet sport. Perception, 28, 307-320.

Carello, C., Thuot, S., \& Turvey, M. T. (2000). Ageing and the perception of a racket's sweet spot. Human Movement Science, 19, $1-20$.

Cross, R., \& Bower, R. (2006). Effects of swing-weight on swing speed and racket power. Journal of Sports Sciences, 24(1), 2330. doi:10.1080/02640410500127876

Davids, K., \& Araújo, D. (2010). The concept of 'Organismic Asymmetry' in sport science. Journal of Science and Medicine in Sport, 13(6), 633-640. doi:10.1016/j.jsams.2010.05.002

Davids, K., Bennett, S. J., \& Beak, S. (2002). Sensitivity of children and adults to haptic information in wielding tennis rackets. In $\mathrm{K}$. Davids, G. J. P. Savelsbergh, S. J. Bennett, \& J. Van der Kamp (Eds.), Interceptive actions in sport: Information and movement (pp. 195-211). London: Routledge.

Davids, K., Renshaw, I., \& Glazier, P. (2005). Movement models from sports reveal fundamental insights into the coordination process. Exercise and Sport Science Reviews, 33, 36-42. doi:0091-6331/ $3301 / 36-42$

Edelman, G. M., \& Gally, J. A. (2001). Degeneracy and complexity in biological systems. Proceedings of the National Academy of Sciences of the United States of America, 98(24), 13763-13768. doi:10.1073/pnas.231499798

Elliott, B. C., Baker, J., \& Foster, D. (1993). The kinematics and kinetics of the off-drive and on-drive in cricket. Australian Journal of Science and Medicine in Sport, 25, 48-54.

Fajen, B. R., Diaz, G., \& Cramer, C. (2011). Reconsidering the role of movement in perceiving action-scaled affordances. Human Movement Science, 30, 504-533. doi:10.1016/j.humov.2010.07.016

Fajen, B. R., Riley, M. A., \& Turvey, M. T. (2009). Information, affordances, and the control of action in sport. International Journal of Sport Psychology, 40, 79-107.

Field, A. (2009). Discovering statistics using SPSS (3rd ed.). London: SAGE publications.

Fisher, S., Vogwell, J., \& Ansell, M. P. (2006). Measurement of hand loads and the centre of percussion of cricket bats. Proceedings of the Institution of Mechanical Engineers, Part L: Journal of Materials Design and Applications, 220, 249-258. doi:10.1243/ 14644207JMDA77

Fleisig, G. S., Zheng, N., Stodden, D. F., \& Andrews, J. R. (2002). Relationship between bat mass properties and bat velocity. Sports Engineering, 5(1), 1-8. doi:10.1046/j.1460-2687.2002.00096.x

Gibson, J. J. (1966). The senses considered as perceptual systems. Boston: Houghton Mifflin. 
Gibson, J. J. (1979). The ecological approach to visual perception. Hillsdale: Erlbaum.

Hove, P., Riley, M. A., \& Shockley, K. (2006). Perceiving affordances of hockey sticks by dynamic touch. Ecological Psychology, 18(3), 163-189. doi:10.1207/s15326969eco1803_ 2

Kelso, J. A. S. (1995). Dynamic patterns: The self-organization of brain and behavior. Cambridge: MIT press.

Koenig, K., Mitchell, N. D., Hannigan, T. E., \& Clutter, J. K. (2004). The influence of moment of inertia on baseball/softball bat swing speed. Sports Engineering, 7, 105-117. doi:10.1007/ BF02915922

Michaels, C. F., Weier, Z., \& Harrison, S. J. (2007). Using vision and dynamic touch to perceive the affordances of tools. Perception, 36 (5), 750-772. doi:10.1068/p5593

Müller, S., \& Abernethy, B. (2008). Validity and reliability of a simple categorical tool for the assessment of interceptive skill. Journal of Science and Medicine in Sport, 11(6), 549-552. doi:10.1016/ j.jsams.2007.08.003

Newell, K. M. (1985). Coordination, control and skill. In D. Goodman, R. B. Wilberg, \& I. M. Franks (Eds.), Differing perspectives in motor learning, memory and control (pp. 295-317). Amsterdam: Elsevier Science.

Newell, K. M. (1986). Constraints on the development of coordination. In M. G. Wade \& H. T. A. Whiting (Eds.), Motor development in children: Aspects of coordination and control (pp. 341-360). Dordrecht: Martinus Nijhoff.

Pinder, R. A., Davids, K., Renshaw, I., \& Araújo, D. (2011). Manipulating informational constraints shapes movement reorganization in interceptive actions. Attention, Perception \& Psychophysics, 73, 1242-1254. doi:10.3758/s13414-011-0102-1

Pinder, R. A., Renshaw, I., \& Davids, K. (2009). Informationmovement coupling in developing cricketers under changing ecological practice constraints. Human Movement Science, 28, 468479. doi:10.1016/j.humov.2009.02.003

Rein, R., Davids, K., \& Button, C. (2009). Adaptive and phase transition behavior in performance of discrete multi-articular actions by degenerate neurobiological systems. Experimental Brain Research, 201(2), 307-322. doi:10.1007/s00221-009-2040-x

Renshaw, I., Oldham, A. R. H., Davids, K., \& Golds, T. (2007). Changing ecological constraints of practice alters coordination of dynamics interceptive actions. European Journal of Sport Science, 7(3), 157-167. doi:10.1080/17461390701643026
Shockley, K., Carello, C., \& Turvey, M. T. (2004). Metamers in the haptic perception of heaviness and moveableness. Perception \& Psychophysics, 66(5), 731-742.

Shockley, K., Grocki, M., Carello, C., \& Turvey, M. T. (2001). Somatosensory attunment to the rigid body laws. Experimental Brain Research, 136, 133-137. doi:10.1007/s002210000589

Smith, M. R. H., Flach, J. M., Dittman, S. M., \& Stanard, T. (2001). Monocular optical constraints on collision control. Journal of Experimental Psychology, 27(2), 395-410. doi:10.1037//00961523.27.2.395

Solomon, H. Y., \& Turvey, M. T. (1988). Haptically perceiving the distances reachable with hand-held objects. Journal of Experimental Psychology: Human Perception and Performance, 14(3), 404-427. doi:10.1037/0096-1523.14.3.404

Southard, D., \& Groomer, L. (2003). Warm-up with baseball bats of varying moments of inertia: Effect on bat velocity and swing pattern. Research Quarterly for Exercise and Sport, 74(3), 270-276.

Stretch, R., Bartlett, R., \& Davids, K. (2000). A review of batting in men's cricket. Journal of Sports Sciences, 18(12), 931-949. doi:10.1080/026404100446748

Stretch, R., Buys, F., Du Toit, E., \& Viljoen, G. (1998). Kinematics and kinetics of the drive off the front foot in cricket batting. Journal of Sports Sciences, 16, 711-720. doi:10.1080/026404198366344

Turvey, M. T. (1996). Dynamic touch. American Psychologist, 51(11), 1134-1152. doi:10.1037/0003-066X.51.11.1134

Turvey, M. T., Burton, G., Amazeen, E. L., Butwill, M., \& Carello, C. (1998). Perceiving the width and height of a hand-held object by dynamic touch. Journal of Experimental Psychology: Human Perception and Performance, 24(1), 35-48. doi:10.1037/00961523.24.1.35

Wagman, J. B., \& Carello, C. (2001). Affordances and inertial constraints on tool use. Ecological Psychology, 13(3), 173-195. doi:10.1207/S15326969ECO1303 1

Wagman, J. B., \& Carello, C. (2003). Haptically creating affordances: The user-tool interface. Journal of Experimental Psychology: Applied, 9(3), 175-186. doi:10.1037/1076-898X.9.3.175

Weast, J. A., Shockley, K., \& Riley, M. A. (2011). The influence of athletic experience and kinematic information on skill - relevant affordance perception. The Quarterly Journal of Experimental Psychology, 64(4), 689-706. doi:10.1080/17470218.2010.523474

Woolmer, B., Noakes, T., \& Moffett, H. (2008). Bob Woolmer's art and science of cricket. London: New Holland. 